\title{
Using N-body simulations to understand the chemo-dynamical evolution of the inner Milky Way
}

\section{E. Athanassoula}

Aix Marseille Univ, CNRS, LAM, Laboratoire d'Astrophysique de Marseille, Marseille, France email: lia@lam.fr

\begin{abstract}
I present examples of how chemo-dynamical N-body simulations can help understanding the structure and evolution of the inner Galaxy. Such simulations reproduce the observed links between kinematics, morphology and chemistry in the bar/bulge region and explain them by the self-consistent cohabitation of a number of components. Galactic archaeology, applied to simulation snapshots, explains the sequence in which the stars of the various components were formed. The thick disc stars form earlier than those of the thin disc and in a much shorter time scale. The bar in the thick disc is horizontally thicker than that of the thin disc and has a different vertical morphology. The Galaxy's inner disc scalelength is much smaller than what is expected from nearby galaxies of similar stellar mass.
\end{abstract}

Keywords. Galaxy: bulge - Galaxy: stellar content - Galaxy: structure - Galaxy: kinematics and dynamics - Galaxy: evolution - galaxies: structure — galaxies: kinematics and dynamics — galaxies:spiral — galaxies:evolution — galaxies:evolution

\section{Introduction}

The unprecedented quality and quantity of the data on the Milky Way (MW), from Gaia and from the accompanying ground-based surveys (BRAVA, RAVE, SEGUE, ARGOS, APOGEE, LAMOST, Gaia-ESO, VVV-ESO, GALAH, WEAVE, 4MOST, etc.) has instigated a large number of N-body simulations, which include gas, star formation, feedback and cooling, and, in a few cases, chemical evolution. It is not possible to discuss them all in this review, so I focus on some specific recent results, thereby forsaking any attempt for completeness. Furthermore, I do not discuss here any cosmological simulations, since they are the subject of Scannapieco's review (this volume), and I barely touch on results on the solar neighbourhood, as they are extensively discussed e.g. in the reviews by Minchev (2017) and by Martig (this volume).

For all the figures and analyses made specifically for this review I used the survey of several hundred high resolution simulations initiated with the work described in Athanassoula et al. (2016) (hereafter A16; see also Rodionov et al. 2017 and Athanassoula et al. 2017, hereafter A17) and to which I will refer here as the ARPL simulations. Each of these starts off with two idealised but cosmologically motivated protogalaxies, composed initially only of dark matter and hot gas, and set on an orbit which will bring them sooner or later to merge. Their mass ratio is such as to lead to a major, or intermediate merger. Stars are not present in these initial conditions, but start forming well before the merging, creating a protodisc in each protogalaxy. The merging destroys these two discs, while the two dark matter and the two gaseous haloes merge. A new disc is then formed gradually - inside out horizontally and outside in vertically - from accreting halo gas. By including in the formation scenario such a merger (which, in current cosmological models, is expected to be part of the formation history of a considerable fraction of 
MW-like galaxies), these simulations become more realistic, and can naturally account for components such as a classical bulge or a thick disc with realistic properties and chemistry distinct from that of the thin disc. It also explains better the observations, as e.g. the kinematics of low metallicity stars (Ness et al. (2013) and A17), the formation of the various types of radial density profiles etc. The large number of ARPL simulations available also allows a more homogeneous approach to many important issues on the structure and evolution of MW-type galaxies.

Last but not least, I discuss some specific comparisons with external galaxies, because it should not be forgotten that our Galaxy is one of many, and thus could not have properties at odds with those of other disc galaxies of similar type and mass.

\section{Stellar density distribution}

Observations have shown that the vast majority of the stars in MW-type spiral galaxies lie in a disc component which has an exponential radial density profile with, or in a few cases without, a break separating the inner from the outer disc. Our Galaxy is no exception. These structures, as well as their properties as a function of lookback time and stellar age, have been well reproduced by simulations (e.g. by Radburn-Smith et al. 2012, Aumer \& White 2013, Martig et al. 2014, A16, Aumer et al. 2016, references therein, and as is shown in more detail in ongoing work in collaboration with Peschken et al.).

The left panel of Fig. 1 shows the inner disc scalelengths, $R_{i n}$, of the galaxies in the Laine et al. (2014) sample from the $\mathrm{S}^{4} \mathrm{G}$ (Sheth et al. 2010, Salo et al. 2015) and NIRS0S (Laurikainen et al. 2011) samples, as a function of their stellar mass $\left(M_{*}\right)$. The rectangle outlines a region in which the MW values should be located, i.e. with an $M_{*}$ of $4-7 \times$ $10^{10} \mathrm{M}_{\odot}$ and an $R_{i n}$ of $2-3 \mathrm{kpc}$. Note that for the sample in Fig. 1. only 7 out of 49 external galaxies with a MW-like stellar mass (or 4 out of 30, if the mass limit is $5-7$ $\times 10^{10} \mathrm{M}_{\odot}$ ), have the required $R_{\text {in }}$, all the remainder having considerably larger values, i.e. our Galaxy has a short $R_{\text {in }}$ for its mass.
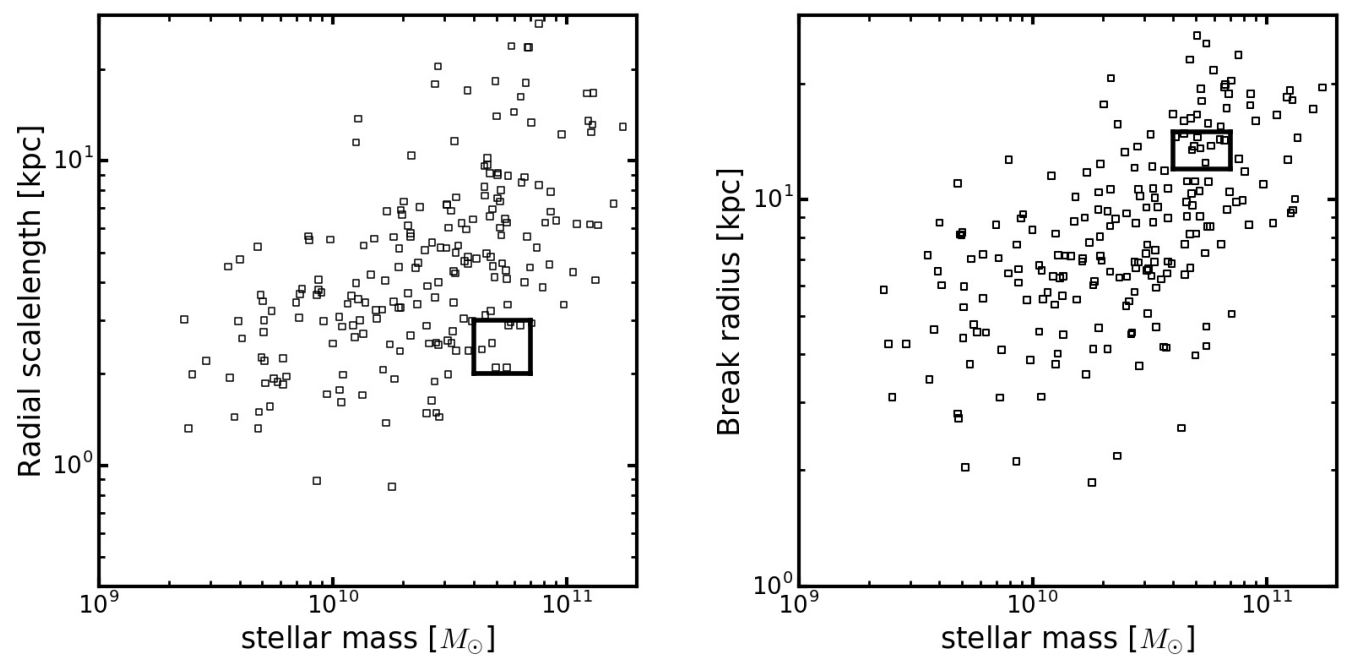

Figure 1. Left: Inner disc scalelength of galaxies from the $S^{4} G$ and NIRSOS samples as a function of their stellar mass. The open rectangle outlines the region where the values of the MW are expected to lie. Right: Similar plot, but now for the break radius. 
The MW break radius is of course less accurately known than the inner disc scalelength, but still, the right panel of Fig. 1 1 shows clearly that it is much nearer to that of the average external galaxy. Thus the MW inner scalelength is much smaller than expected both for its $M_{*}$ and its radial extent, the latter measured by the break radius.

Swing amplification (Toomre 1981) - as applied to external galaxies (Athanassoula et al. 1987) and using the parameter values given by Bland-Hawthorn \& Gerhard (2016) predicts that at the solar region the spiral arm multiplicity $(m)$ is around $4-5$. This is in agreement with the observations of Georgelin \& Georgelin (1976), and does not disagree with an $m=2$ in the inner parts. Although a number of such disc galaxies are known (e.g. de Vaucouleurs \& Pence 1978, Efremov 2011 and references therein), they are still a minority, compared to other barred galaxy morphologies. This again implies that the MW is similar to some external galaxies, but still far from the average barred galaxy. Note that NGC 5012 - which is the only one external galaxy from the sample in Fig. 1 with an $M_{*}$, an $R_{i n}$ and a break radius compatible with those of the MW - has also 4 5 spiral arms outside the central region.

\section{Galactic archaeology}

Galactic archaeology uses properties (such as kinematics, spatial distribution and surface chemical abundances) of present day stars, to understand the formation and evolution of our Galaxy. The stars can be divided in groups according to their age (or its proxy) and the differences between the properties of the various groups is used to outline the Galaxy's formation and evolution.

To test Galactic archaeology techniques, A16 applied them to the final snapshot of one of their simulations and divided the stars into groups as a function of their age. The dividing times were chosen to be landmark times of the evolution. The two main such times are the beginning and the end of the merging period, i.e. of the time span during which the two protogalaxies merge. Although these two times can not be precisely calculated, all reasonable estimates will give the same qualitative results and very similar quantitative ones. The end of the merging period can be considered the same as the beginning of the disc formation $\left(t_{b d}\right)$. A16 studied five age groups separately using faceon and end-on morphology, radial and vertical surface density profiles and the circularity of the individual stellar orbits. The latter is defined as the $z$ component of the angular momentum, normalised by the angular momentum of a circular orbit with the same energy (Aumer \& White 2013, A16).

Before the merging period starts, the two protogalaxies are apart, forming their stars in isolation. These stars undergo violent relaxation during the merging and, viewed globally at the end of the simulation, exhibit properties similar to those of a classical bulge or stellar halo. Thus gas-rich major or intermediate mergers can explain the formation of these components, whose stars have formed during the initial stages of the evolution.

After $t_{b d}$, stars form gradually from accreting halo gas and within a thin disc component. Thus the thin disc is formed during the later secular evolution times.

The evolution of stars born in the intermediate times, i.e. during the merging period, is more complex. Most stars forming during the beginning of that period contribute mainly to the classical bulge and stellar halo, but most stars born in the later stages of the merging period contribute to the thick disc. There is no real discontinuity at any time, but a continuous shift from spheroids to a thick disc component and then to a thin disc.

Simulations such as in A16 show how the various stages of formation and evolution interconnect, shifting from merger-driven to secularly-driven regimes. They also argue that the formation is generally from inside out horizontally, and from outside in vertically. 
Thus, the thick disc forms first and in a relatively short time scale of the order of a Gyr, while the thin disc starts forming later and continues its growth for a much more extended period of time, of the order of $10 \mathrm{Gyr}$. All this is in agreement with the observed bimodality in e.g. the $[\alpha / \mathrm{Fe}]-[\mathrm{Fe} / \mathrm{H}]$ relation, and could provide an explanation of the existence of two distinct trends. Thus this galaxy formation picture leads naturally to the star formation history necessary for the chemical evolution model of Chiappini et al. $(1997)$.

Note also that some of the stars born in the thin disc sufficiently early on will be heated by encounters with e.g. spiral arms, giant molecular clouds, or globular clusters and thus can become members of the thick disc, as will be discussed in more detail elsewhere.

\section{Bars}

\subsection{General}

Bars drive considerable secular evolution in disc galaxies, via angular momentum and mass redistribution. The former takes place via exchanges between the various resonances in the disc and halo (Athanassoula 2002, 2003, or for a general review Athanassoula 2013 and references therein). As most disc galaxies, the MW has a bar. Its length, however, is shorter than what would be expected for galaxies with a MW-like $M_{*}$. This is not necessarily a new particularity of the MW, but may simply be due to the fact that the $R_{i n}$ of the MW is small, as discussed in Sect. 2. Thus the bar length, as measured in kpc, would indeed be smaller than expected, but, when measured in inner disc scalelengths, would be much nearer to expectations. This will also be the case for the resonant radii, so that the radial extent of the region which is strongly influenced by the bar would be similar to that of other barred galaxies, when measured in disc scalelengths.

\subsection{A bar in the thick disc}

Nearly 35 years ago, using N-body simulations with the resolution allowed by the computers at the time, Athanassoula (1983) predicted that the bar shape depends on the stellar velocity dispersion; cold discs forming thinner bars than hot ones. She also showed that if there are two stellar populations, of different stellar velocity dispersions, the cold one will create a thin bar, while the hot one will create a thicker bar with the same length and pattern speed. These predictions have been confirmed by a number of more recent and technically superior simulations (Athanassoula 2003, Bekki \& Tsujimoto 2011, A16, Debattista et al. 2017, Fragkoudi et al. 2017). It is thus expected that, in the MW, both the thick and the thin disc would grow a bar. Whether these can be considered as two separate bars, dynamically locked to each other, or one single bar where stars of different ages outline different morphologies, depends on whether one believes there is a clear separation between the two discs, or a more or less continuous transition.

Most of the above works have very idealised initial conditions, in which the thin and the thick disc populations are present and separated already in the initial conditions of the simulations. It is then trivial to distinguish between the two bars and to find to which bar a given particle belongs. This, however, is not the case in observations, where the two populations have to be distinguished e.g. by their age, or their metallicity. Simulations with less idealised initial conditions, where the thin and the thick discs do not pre-exist in the initial conditions, but form during the evolution, are closer to observations. This is the case with the ARPL simulations. These present a number of advantages. More specifically, gas is accreted from the galactic halo and forms stars, so that the age of each star is trivially calculated all through the simulation and there is no need of proxies. Moreover, the thick disc forms naturally in this scenario and has properties which agree 
with observations (A16). In fact, the third and fourth panels (from the left) of Fig. 4 in A16 have already shown that the thick disc population, viewed face-on at the end of the simulation, has a central thick bar of oval shape, which viewed side-on (i.e. edge-on, with the line of sight along the bar minor axis) has a boxy shape. On the contrary, the younger stars, born in the thin disc after those in the thick disc, show face-on a much thinner bar and side-on an X shape. For further discussion on this see Sect. 3.9 of A16.

The example discussed in A16 has a short and relatively weak bar. I will extend their analysis here using a simulation and snapshot with a longer and stronger bar, which increases the $\mathrm{S} / \mathrm{N}$ ratio and illustrates better the effects I wish to show. I follow the same procedure as in A16, but contrary to it I include in the decomposition only stars which at the end of the simulation are part of the bar, as found according to their location and kinematics. The result is shown in Fig. 2, while a colour version of this plot can be seen in slide 20 of my oral presentation (https://iaus334.aip.de/pdf-of-the-talks/). In the latter the colour represents age, the oldest in each group in red and the youngest in blue. It is clear that the oldest bar population - which contains the stars formed in the later part of the merging - are distributed face-on within a thick oval shape, and edge-on extend to large distances from the equatorial plane, outlining a boxy-like shape. On the contrary, the younger ones, born during the thin disc formation time, are distributed in a much thinner bar shape face-on and more X-like shape edge-on. This is in good agreement with what is expected from previous work (see in particular A16).

The youngest stars, of age less than a Gyr, form the thinnest of all distributions, both horizontally and vertically. It is quite possible that these stars may form a vertically superthin bar as the one discussed by Wegg et al. (2015). A full appraisal of this suggestion, however, will necessitate a baryonic softening of the order of at most $10 \mathrm{pc}$ and better around $5 \mathrm{pc}$, while most of the ARPL simulations have a softening of $25 \mathrm{pc}$, which,

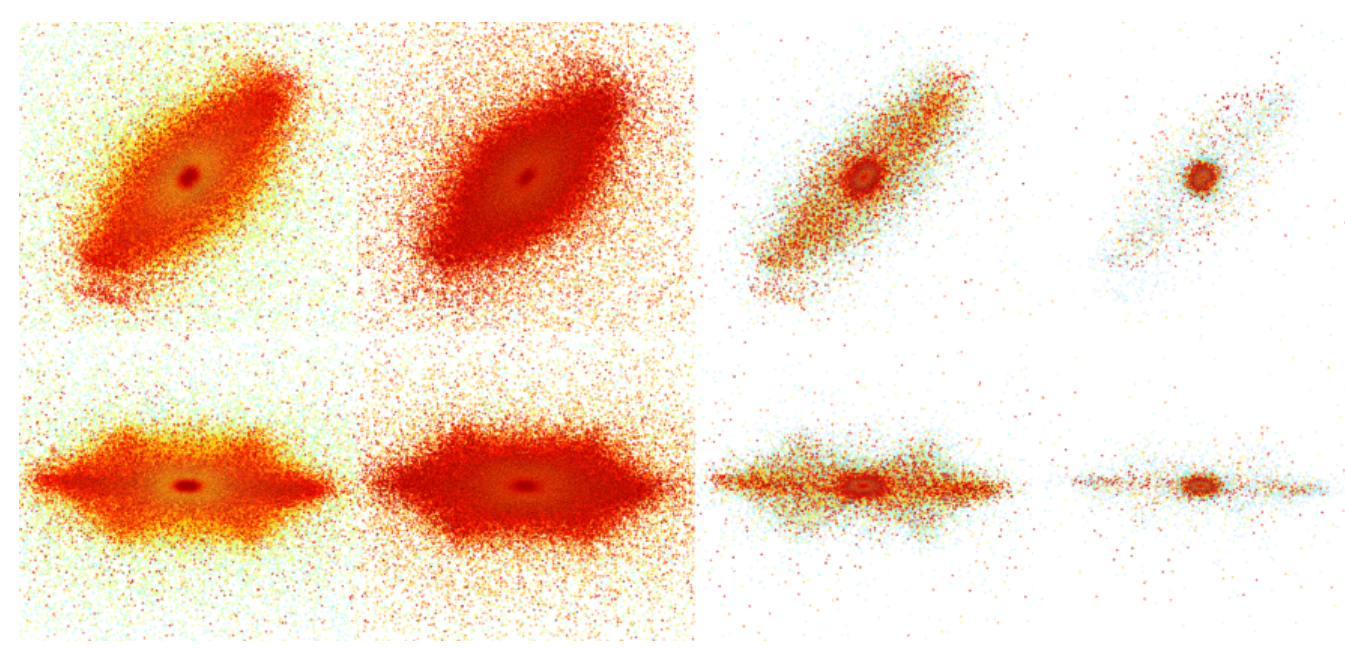

Figure 2. Comparison of the bar morphology for stars in different age ranges (see text). The upper panels in each column give the face-on view with the bar roughly on the diagonal, and the bottom ones the side-on view. The leftmost column of panels includes bar stars of all stellar ages. For the remaining three columns of panels, the stellar age decreases from left to right. The second column from the left includes mainly the stars in the thick disc and the third and fourth columns mainly the stars in the thin disc. Most of them are in the third panel, except for the youngest (not older than one Gyr) which are in the rightmost panel. Note the strong difference in morphology between the bars of different age stars. 
although considerably smaller than most other simulations addressing this question, is still larger than what is required.

Hence these simulations show that the thick disc bar morphology is different from that of the thin disc, resembling, face-on, more a fat oval. They also do not have ansae and the thick disc in general shows no spirals, unless of very low amplitude (A16). These predictions should be testable for our Galaxy using data from Gaia and the accompanying ground-based surveys.

\section{Coupling chemical evolution with kinematics and morphology in the bar/bulge region}

The high quality data delivered by the spectroscopic surveys accompanying Gaia brought a lot of information on the element abundances of MW stars. This can be used to set further constraints on the models and in particular on the new N-body simulations.

A number of the early chemo-dynamical simulations included no gas component and thus no information on stellar ages and no possibility of modelling the chemical evolution. The metallicities and abundances were replaced by proxies. This can be hazardous, but still such codes have been often used and allowed some interesting initial insights. With the advent of N-body codes with a full hydrodynamic treatment, i.e. including star formation, feedback and cooling, a few codes made the extra steps necessary to include chemical evolution. This brought much tighter constraints on the models, opening up a whole new realm of possibilities. I will focus here only on the bar/bulge region.

Observations clearly showed that the metallicity, kinematics and spatial distribution of the stars are strongly coupled to each other. Babusiaux (2016), using data from a number of sources, plotted the line of sight velocity dispersion $(\sigma)$ as a function of the absolute value of the latitude separately for low metallicity $(\mathrm{LM},-1.0<[\mathrm{Fe} / \mathrm{H}]<-0.5)$ and high metallicity $(\mathrm{HM}, 0 .<[\mathrm{Fe} / \mathrm{H}]<0.5)$ stars, neglecting the intermediate ones (IM), and finds they have a quite different behaviour. For the LM stars, $\sigma$ is roughly constant, showing no dependence on latitude. On the contrary, for the HM stars $\sigma$ shows a strong decrease with increasing latitude reaching much smaller values of $\sigma$ than the LM at high latitudes. Using one of the very high resolution ARPL simulations, A17 reproduced and discussed this behaviour (see left panel of Fig. 1 in A17).

In similar, but more in depth and more extended studies, Ness et al. (2013, their Fig. 6) using the ARGOS sample (Freeman et al. 2013) and Zasowski et al. (2016, their Fig.

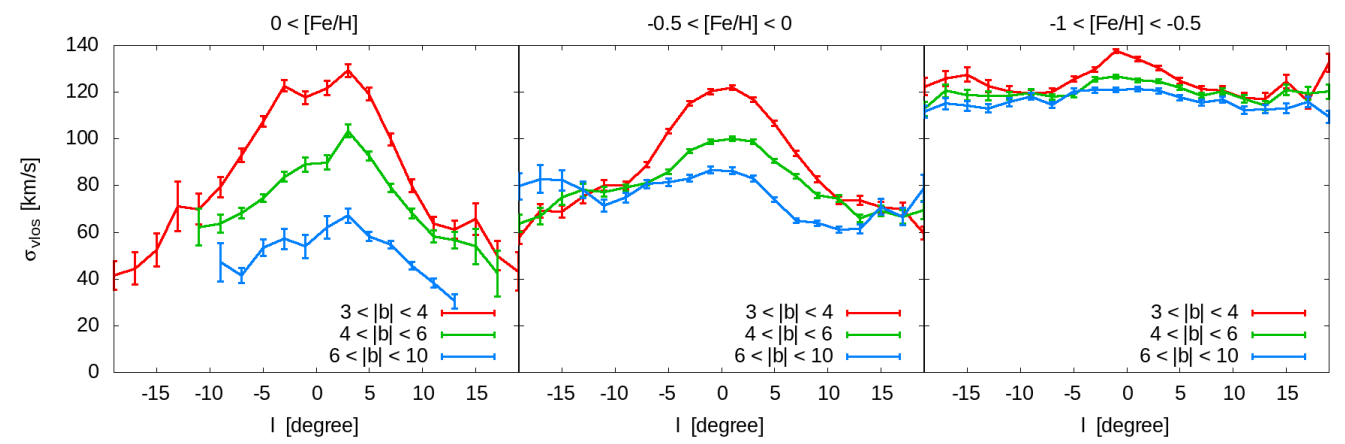

Figure 3. Link between the metallicity and kinematics: Line-of-sight velocity dispersion as a function of longitude. Stars are divided into groups as a function of their latitude and of their metallicity (HM to the left, LM stars to the right, and IM in the middle panel). Reproduced from Fig. 2 of A17. 
9) using APOGEE data (Majewski et al. 2017) showed the link between the metallicity and the kinematics, not only as a function of latitude, but also as a function of longitude. At low latitudes the $\sigma$ of HM stars shows a clear, strong maximum at zero longitude, declining sharply with increasing absolute value of longitude. Still for HM stars, but now at larger distances from the Galactic equatorial plane, $\sigma$ shows lower values and a much shallower decrease with absolute longitude. The LM stellar population has higher values of $\sigma$ and much less of a decrease than the HM one. Populations of intermediate metallicity have also intermediate kinematics. These qualitative behaviours are found in both the ARGOS and the APOGEE data. With their very high resolution simulations, A17 reproduce all these trends and explain them by linking both the metallicity and the kinematics to the stellar origins. Thus, stars born before or during the merging time will have low metallicities and high velocity dispersions. As mentioned in Sect. 3 . they constitute the stellar halo, the classical bulge (whenever present) and the thick disc. On the other hand, stars born during the secular evolution time will form mainly in the thin disc and will have higher metallicities and lower $\sigma$ values. Thus there is a link not only between kinematics and metallicity, but also between these quantities and morphology. Indeed, metallicity-defined populations are found to have strikingly different spatial distributions. The LM stars are distributed in a spheroidal-like shape highly flattened in the vertical direction, and including a thick, low-density disc. They do not show any, or very little, X-shaped structure, while the HM stars outline only the X-shape and a clear underlying disc component (Figs. 3 and 4 of A17), all in good agreement with observations.

Note that the simulations reproduce very well qualitatively all the observed trends. It is not useful or even appropriate to make quantitative comparisons, as non-negligible quantitative differences exist even between the afore mentioned observational studies, presumably due to the differences in the selection criteria of the two samples. Also such differences can be due to small differences in the chemical models (A17). The most important reason, however, is simply that we do not yet have a simulation which gives a sufficiently good match to al the MW structural and dynamical properties.

\section{A few concluding words}

I briefly described a few examples demonstrating how useful high quality N-body simulations can be in explaining the formation, structure and evolution of MW-like galaxies. Such simulations should be of high resolution, and include gas, star formation, feedback and cooling, and preferably be coupled to a chemical evolution code, thus giving results on metallicity and element abundances and allowing comparisons with the results of the numerous ground-based surveys accompanying Gaia. It will thus be possible to set very tight constraints on the models, particularly for the formation and evolution of the various stellar populations and components. The examples discussed here, and/or in the oral version (https://iaus334.aip.de/pdf-of-the-talks/), include galactic archaeology examples leading to a thick disc formation scenario, the formation and evolution of bars, the distinction between bars forming in the thin and the thick disc, the role of the halo in the evolution, the formation and evolution of exponential radial density profiles with and without breaks, and, last but not least, the links between the kinematics, metallicity and morphology of the various stellar populations in the bar/bulge region. Obviously this list is far from exhaustive, but is only meant to give a flavour of the available possibilities.

It is also important to keep in mind that our Galaxy is just one disc galaxy among many others. It is unique only in the sense that, as we are in it, we can observe individual stars at a level which can not yet be achieved for most external galaxies. However, it should not 
be unique, either regarding its formation and evolution, or regarding its structure and contents. Note that numerous external galaxies have been well observed, thus providing invaluable statistical knowledge, while being exempt of the geometrical problems which we have in the MW due to our location in it. Thus a lot has to be gained by close collaborations between people working on our Galaxy and those working on external galaxies.

\section{Acknowledgements}

I thank the organisers for inviting me to this interesting and inspiring meeting. I thank the CNES for financial support and my collaborators A. Bosma, J.C. Lambert, N. Peschken, N. Prantzos, and S. Rodionov, for many useful discussions and/or help with technical aspects. I acknowledge use of HPC resources from GENCI/TGCC/CINES and from Mesocentre of Aix-Marseille-Université.

\section{References}

Athanassoula, E. 1983, in E. Athanassoula (ed.), Internal kinematics and dynamics of galaxies, Dordrecht: D. Reidel Publishing Co., p. 243

Athanassoula, E. 2002, ApJ, 569L, 83

Athanassoula, E. 2003, MNRAS, 341, 1179

Athanassoula, E. 2013, in J. Falcón-Barroso and J. H. Knapen (eds.), Secular Evolution of Galaxies, Cambridge, UK: Cambridge University Press, p. 305

Athanassoula, E., Bosma, A. \& Papaioannou, S. 1987, A\& A, 179, 23

Athanassoula, E., Rodionov, S., Peschken, N., \& Lambert, J.C. 2016, ApJ, 821, 90 (A16)

Athanassoula, E., Rodionov, S., \& Prantzos, N. 2017, MNRAS, 467L, 46 (A17)

Aumer, M., \& White, S. 2013 MNRAS, 428, 1055

Aumer, M., Binney, J., \& Schönrich, R. 2016, MNRAS, 459, 3326

Babusiaux, C. 2016, Publ. Astr. Soc. Autralia, 33, 26

Bekki, K., \& Tsujimoto, T. 2011, MNRAS, 416, 60L

Bland-Hawthorn, J., \& Gerhard, O. 2016, ARA\&A, 54, 529

Chiappini, C., Matteucci, F., \& Gratton, R. 1997, ApJ, 477, 765

Debattista, V., Ness, M., Gonzalez, O. et al. 2017, MNRAS, 469, 1587

Efremov, 2011, Astr. Rep., 55, 108

Fragkoudi, F., Di Matteo, P., Haywood, M. et al. 2017, arXiv170400734

Freeman, K.C., Ness, M., Wylie-de-Boer, E. et al. 2013, MNRAS, 428, 3660

Georgelin Y. M., \& Georgelin Y. P. 1976, A\& A, 49, 57

Laine, J., Laurikainen, E., \& Salo, H. 2014, A\& A, 596, A25

Laurikainen, E., Salo, H., Buta R., \& Knapen, J. 2011, MNRAS, 418, 1452

Majewski, S., Schiavon, R,, Frincaboy, P. et al. 2017, AJ, 154, 94

Martig, M., Minchev, I., \& Flynn, C. 2014, MNRAS, 442, 2474

Minchev, I. 2017, arXiv170107034

Ness, M., Freeman, K.C., Athanassoula, E. et al. 2013, MNRAS, 432, 2092

Radburn-Smith, D. J., Roskar, R., Debattista, V. P., et al. 2012, ApJ, 753, 138

Rodionov, S., Athanassoula, E., \& Peschken, N. 2017, A\&A, 600, A25

Salo, H., Laurikainen, E., Laine, J., et al. 2015, ApJS, 219, 4

Sheth, K., Regan, M., Hinz, J. L., et al. 2010, PASP, 122, 1397

Toomre, A. 1981, in Fall, S. M. and Lynden-Bell, D. (eds.), The structure and evolution of normal galaxies, Cambridge and New York: Cambridge University Press, p. 111

de Vaucouleurs, G., \& Pence, W. D. 1978, AJ, 83, 1163

Wegg, C., Gerhard, O., \& Portail, M. 2015, MNRAS, 450, 4050

Zasowski, G., Ness, M., García Pérez, A., et al. 2016, ApJ, 832, 132 